Malawi). Der Autor räumt selbst ein, daß das beschränkte Untersuchungsfeld ebenso wie die Knappheit der erhältlichen Daten die Aussagekraft seiner Thesen begrenzt.

Diese Thesen knüpfen an zwei Punkten an, für die solche Daten noch am ehesten zugänglich sind. Zum einen glaubt er eine Beziehung zu sehen zwischen der steigenden und fallenden Häufigkeit von Parlamentssitzungen einerseits und Militärcoups andererseits; fallende Trendkurven für die Coup-Staaten Ghana, Nigeria und Uganda sollen das untermauern (S. $17 \mathrm{ff}$.). Außerdem soll auch die $\mathrm{Zu}$ - oder Abnahme von Wahlkreisen, in denen sich mehrere Kandidaten zur Wahl stellen, etwas über die Tendenz zum Militärputsch aussagen. Wenngleich der Verfasser betont, er wolle auf diese Weise nicht den Grad an erreichter Demokratie quantifizieren (S. 30), so kann man sich doch des Eindrucks nicht erwehren, hier werde der Versuch unternommen, die Wahrscheinlichkeit einer Machtübernahme durch die Armee mit ähnlich seismologischer Genauigkeit vorherzusagen wie einen Vulkanausbruch. Zwar ist dem Autor zuzugeben, daß solche Krisen in Entwicklungsstaaten nicht einfach als mysteriöse "Naturereignisse“ gesehen werden dürfen. Aber er hält in wenig überzeugender Weise das Symptom „mangelnde parlamentarische Aktivität" für eine, wenn auch nicht die einzige Ursache der fallenden Systemstabilität. Dabei überschätzt der Verfasser die legitimierende Funktion von Parlamenten gerade in Einparteienstaaten wie Kenia, Sambia, Tansania und Malawi erheblich.

Alexander Dix

Kroniek van Afrika 1975/1, No. 4

Special Issue on Disparity between Law and Social Reality in Africa Afrika-Studiecentrum, Leiden

Die Frage nach der Effektivität von Rechtsnormen ist eines der zentralen Themen der überseeischen Rechtsvergleichung, wobei man es allerdings vermeiden sollte, einen absoluten Gegensatz zwischen ineffektivem Recht in Entwicklungsländern und effektivem Recht in Industriestaaten zu machen, da dies den rechtssoziologischen Erkenntnissen über die begrenzte Bedeutung rechtlicher Normen für menschliches Verhalten in allen Gesellschaftsordnungen widersprechen würde. Die holländische Zeitschrift „Kroniek van Afrika“ hat diesem Thema ein Sonderheft gewidmet, in dem nach einer kurzen rechtssoziologischen Einleitung über die Problemstellung bei Untersuchungen zur Effektivität des Rechts (Kurczewski/ Podgórecki) anthropologische Fallstudien den Schwerpunkt bilden. Dabei scheint der Titel des Sonderheftes "Disparity between Law and Social Reality in Africa“ insofern nicht ganz richtig gewählt zu sein, als es in den behandelten Konfliktfällen weniger um einen Zusammenprall von Recht und Wirklichkeit als um den unterschiedlicher Rechtsnormen (endogener und europäischer) geht. Die Fallstudien beschäftigen sich überwiegend mit Konflikten in sozialen Gruppen, die unter der komplexen und pluralistischen Organisation afrikanischer Rechtsordnungen besonders zu leiden haben, nämlich partiell in den "modernen“ Sektor integrierte Nicht-Elite-Gruppen. Während die Eliten mit europäischen Rechtsmodellen leben können und (seltener werdende) intakte afrikanische Gesellschaften solche Modelle ignorieren, haben solche Gruppen die volle Kraft des Konflikts auszuhalten. Das führt dann dazu, daß es gegen als Unrecht empfundene (und in 
einer intakten Stammesgesellschaft zweifellos auch sanktionierte) Handlungen keinen rechtlichen Schutz gibt (Harrell-Bond; Lowy), oder daß Konflikte Lösungen finden, die das Rechtsbewußtsein aller Beteiligten verletzen (Rijnsdorp). Ein anderer Fall (van Rouveroy) zeigt, wie der Rechtspluralismus es Afrikanern, die sich im westlichen Recht auskennen, erlaubt, ihre weniger gebildeten Stammengenossen übers Ohr zu hauen. Auch die Diskussion von Eheproblemen im städtischen West-Nigeria (Aig-Ojehomon-Ketting) beleuchtet die Schwierigkeiten und Konflikte einer Übergangssituation, die mit den Begriffen "modern" und "traditionell“ nicht einmal mehr idealtypisch zu erfassen ist. Verdier zeigt in einem abschließenden Aufsatz, wie die Grundlagen für die heutigen Probleme nicht zuletzt in Mißverständnissen der afrikanischen Gesellschaftsstruktur in der Kolonialzeit ihren Ursprung haben. Ein Besprechungsaufsatz über die (nicht sehr zahlreichen) Arbeiten zum Thema (Gerold-Scheepers) vervollständigt das Heft.

Brun-Otto Bryde

\section{Ben O. Nwabueze}

\section{Constitutionalism in the Emergent States}

With a foreword by S. A. de Smith

C. Hurst \& Co. in association with Nwamife Publishers, London/Enugu 1973, XII, $316 \mathrm{~S}$.

Diese Untersuchung hat einen sehr grundsätzlichen Gegenstand und Anspruch, wenngleich ihr Titel in seiner Allgemeinheit etwas irreführend ist. Der Verfasser, ein nigerianischer Rechtswissenschaftler, befaßt sich in erster Linie mit der Verfassungsentwicklung in den Commonwealth-Staaten Afrikas, wobei er Nigeria wiederholt Modellcharakter beimißt. Die Entwicklung in den frankophonen Staaten wird weniger intensiv behandelt und nur hin und wieder zum Vergleich herangezogen. Grundsätzlich ist dieser Untersuchungsgegenstand deshalb, weil die Entwicklungen in den einzelnen Staaten, insbesondere in Nigeria, stets auf ihre Verallgemeinerungsfähigkeit hin geprüft werden; der Autor unternimmt den Versuch einer Verfassungslehre für die afrikanischen Entwicklungsstaaten des „Westminster model"-Rechtskreises. Dieser Versuch ist nur zum Teil gelungen, er ist im übrigen durch die tatsächliche Entwicklung und neuere Erkenntnisse weitgehend überholt.

Der Verfasser geht von der klassisch-liberalen Begriffsbestimmung des Konstitutionalismus (1. Kapitel) als Gegenteil von Willkürherrschaft aus (S. 1); die Bedeutung, die er der Antithese Staat - Individuum beimißt, wird erkennbar, wenn er eine unabhängige Judikative als das juristisch bedeutendste Merkmal des modernen Staates bezeichnet (S. 16). Der Begriff des Verfassungsstaates wird noch weiter eingeengt, da der Autor gerade die Normenkontrollkompetenz der ordentlichen Gerichtne für das „Hauptbollwerk“, das "axiomatische Prinzip“ des Konstitutionalismus im Commonwealth und in den USA hält (S. 36 f.). Damit fallen nicht nur diejenigen westeuropäischen Staaten (z. B. die BRD) aus der Kategorie „constitutionalist government" heraus, die nach der Einschätzung des Verfassers mit ihren politisch besetzten, besonderen Verfassungsgerichten schlechtere Erfahrungen gemacht haben als die USA mit dem Supreme Court (S. 20), sondern auch Großbritannien selbst, das Mutterland des Verfassungsstaates. 